\title{
Control of Multiple Underwater Vessels to Converge to a Desired Pattern
}

\author{
Hassan Sayyaadi $^{*}$, Abbas Ghassemzadeh ${ }^{2}$ \\ 1 Professor, Center of Excellence in Hydrodynamics and Dynamics of Marine Vehicles; School of \\ Mechanical Engineering, Sharif University of Technology, Tehran 11155-9567 Iran, \\ Sayyaadi@sharif.edu \\ 2 M.Sc. Student, School of Mechanical Engineering, Sharif University of Technology; \\ abbas_gh68@hotmail.com
}

\begin{tabular}{l} 
ARTICLE INFO \\
\hline Article History: \\
Received: 3 Jun. 2017 \\
Accepted: 15 Mar. 2017 \\
\\
\hline Keywords: \\
Multi agent system \\
Group coordination \\
Swarm \\
Non-Linear Control \\
Vessel Dynamics \\
Autonomous control \\
Decentralized control
\end{tabular}

\section{Introduction}

Group coordination and swarm control are topics that are currently receiving a lot of interest in a variety of research communities, including biology, robotics, communications and sensor networks, artificial intelligence, automatic control etc. Coordinating the motion of a group of relatively simple and inexpensive agents can cover a larger operational area and achieve complex tasks that exceed the abilities of one single agent. Group coordination and swarm control are enabling technologies for applications such as teams of cooperative robots performing demining

\begin{abstract}
The important and hazardous of the rescue mission in oceans and seas, autonomous vessels now are one of the most appropriate applications among others. Due to safety, reliability, and accessibility of smart, Autonomous and Cooperative vessels today has attracted much attention from the industry. Regard to the complication of the mono vessel for different objects, the multiagent system was proposed by the researchers. A group of vessels which are connected to each other through different communication systems like GPS, INS and etc., could easily act their duties in the different situations. Design a strategy controller for a group of underwater vessels with the aid of Lyapanove and Graph theory is addressed in this brief. Realistic dynamics is considered in this paper which is novel things in the fields of control system design to demonstrate the performances of the designed controller. Using realistic dynamics makes it possible to really analyze the behavior of the system and consider all the problems which the systems might be faced in reality. The main features of the proposed controller are the decentralized and scalable controller which convert the controller to be applicable to the different number of agents also in the different situation without any external monitoring and this is while all the previous work were based on the external Control. Due to the realistic agent dynamics, non-holonomic dynamics and constraints of the vessels are considered in the design process Advantages of the proposed controller could be represented as follow: of mestic information is used between vessels. Based on the realistic dynamics diagonal and constant, are considered as non-diagonal and variable. Also to represent the effectiveness of the proposed controllers, MATLAB and SIMULINK are used to simulate the effectiveness of the controller. As the simulation results show, designed controllers perform well on the system and the objective duty is achieved appropriately.
\end{abstract}

operations, monitoring and rescue operation in the different situation.

Due to growth in automation and new generation of unmanned systems, autonomous vessels have got great interested among researchers for many years. Based on the nature of these kinds of systems, Search and rescue operation, surveillance and others are the most application which is multi-agent systems could easily operate in the different situation.

A multi-agent system is a computerized system composed of multiple interacting agents within an environment. Multi-agent systems can be used to 
solve problems that are difficult or impossible for an individual agent or monolithic systems to solve. Most of the researchers are focused on studies on the dynamic and control of multi-agent systems. Therefore, the design of control strategy for this kind of systems is interested in the recent decade. A great deal effort has been directed at developing a centralized and decentralized control strategy for a wide variety of swarm application. In a research, [1], artificial potential and virtual leaders are used for swarm control. Artificial potentials define interaction control forces between neighboring vehicles and are designed to enforce a desired inter-vehicle spacing. A virtual leader is a moving reference point that influences vehicles in its neighborhood by means of additional artificial potentials. Simulations are presented to describe the stability results and give insight into the influence of control parameters on performance.it is important to note that the agents are considered as point masses and fully actuated. Null space-based behavioral control is explained in [2] for formation control of underactuated surface vessels. In this paper, The NSB works in combination with the low-level maneuvering controls of each vessel to take into consideration the dynamics of the fleet. The guidance system has been simulated in a complex mission involving the attainment of a formation while moving through obstacles in presence of sea current; the obtained results show the effectiveness of the proposed method. Behavior-based approaches, widely studied for mobile robotics applications [17], are useful to guide a multi-robot system in an unknown or dynamically changing environment. These approaches give the system the autonomy to navigate in complex environments avoiding low-level path planning, using sensors to obtain instantaneous information of the environment and increasing flexibility of the system. Among the behavioral approaches, seminal works are reported in the papers [18] and [19], while the textbook [17] offers a comprehensive state of the art.

In [3] backstepping method is addressed. This paper discussed that the feedback control scheme is valid as long as the complete dynamics of the followers and their leader is known. Numerical results were presented and the stability of the system was verified. Simulation results verify the theoretical conjecture and expose the flaws in ignoring the dynamics of the mobile robots. In [4] authors use sliding mode tracking control for surface vessels. The control law was developed using the first-order surface in terms of the surge tracking errors and a second-order surface in terms of the sway tracking errors. The vessel's absolute position and orientation were measured using a camera. The motor input voltages were estimated from the controller propeller forces and transmitted to the motors using wireless transmitters and receivers. Several straight-line and circular experiments were successfully performed. Authors in [5] used modified bees algorithm, and in [6] used distance estimation schemes. a set of decentralized control laws, to be incorporated with distance estimation scheme, is introduced to move the robotic swarm in formation from an arbitrary initial position to an arbitrary final position without deforming the formation shape. The robots do not have any global positioning sensors, and they do not communicate with each other. In [7] local adaptive internal model based controllers are presented. Under very mild assumptions local adaptive internal model-based controllers augmented with high gain observers are designed to achieve agreement of the agent outputs The papers [20], [21], [22], [23] considered cooperative control of only a portion of the state vector of each mobile robot and their proposed methods were specialized to a specific class of robotic system. To our knowledge, there is no paper which discusses the cooperative control problem for general nonholonomic agents with limited communication capabilities among neighbors.

In this paper, Lyapunov and graph theory are used for the design of control strategy for swarm formation of multiple vessels in the calm water. This method is based on [8], but cannot be used directly because the model which is selected here, is a real and has more complicated dynamics influences. In other hands, the controller designed in [8] is used for small surface vessels but the model used in this paper is the real underwater vessel, has a total length of 30 meters.

The remaining of the paper is organized as; in section 2 Problem statement of the system are presented and in section 3 dynamic model of the vessel is derived and then coordination transfer is proposed. The mission statement is addressed in section 4 and then cooperative controllers are proposed in section 5 . Finally, simulation results are depicted in section 6.

\section{Problem Statement}

The main focus of this work provides a decentralized strategy for task allocation that makes use of the spatial task information in Autonomous vessels swarms. The difficulty of task allocation increases when the number of the autonomous underwater vessels required by tasks is non-deterministic, the total number of vessels and tasks is high. The aim of developing such a strategy is to facilitate a scalable, fast, and efficient solution. The strategy focuses on utilizing spatial information about tasks and the number of vessels required to achieve efficiency and a decentralized approach to achieve scalability. The problem is to put a group of vessels into an appropriate distance from each other and form them for a special task. The distance from each other is assigned by the controller which is determined based on the size of the vessels and the main task. The tasks are static in time, space, and have constant task size. 


\section{Dynamics Model}

Consider $N$ actuated three DOF underwater vessels which are illustrated in figure 1 . Surge propulsive force is delivered by a propeller and a rudder provides torque capable of affecting yaw. Dynamics model of each vessel can be given in equation 1 to equation 7 appropriately [9] and dynamics equations for the $i$ 'th vessels can be written as:

$$
\begin{aligned}
& \eta_{i}=\left[\begin{array}{l}
x_{i} \\
y_{i} \\
\theta_{i}
\end{array}\right] \text { and } v_{i}=\left[\begin{array}{l}
u_{i} \\
v_{i} \\
r_{i}
\end{array}\right] \\
& \dot{\eta}_{i}=R\left(\theta_{i}\right) v_{i} \\
& R\left(\theta_{i}\right)=\left[\begin{array}{ccc}
\cos \left(\theta_{i}\right) & -\sin \left(\theta_{i}\right) & 0 \\
\sin \left(\theta_{i}\right) & \cos \left(\theta_{i}\right) & 0 \\
0 & 0 & 1
\end{array}\right] \\
& M_{i} \dot{v}_{i}+\left(C_{i}+D_{i}\left(v_{i}\right)\right) v_{i}=\left[\begin{array}{c}
\tau_{1} \\
0 \\
\tau_{3}
\end{array}\right] \\
& M_{i}=\left[\begin{array}{ccc}
m_{i 11} & 0 & 0 \\
0 & m_{i 22} & m_{i 23} \\
0 & m_{i 32} & m_{i 33}
\end{array}\right] \\
& D_{i}=\left[\begin{array}{ccc}
d_{i 11}+d_{i 11}^{n}\left|u_{i}\right| & 0 & 0 \\
0 & d_{i 22} & d_{i 23} \\
0 & d_{i 32} & d_{i 33}
\end{array}\right]
\end{aligned}
$$

Where:

- $\left(x_{i}, y_{i}\right)$ is the location of mass center

- $L_{i}$ is the total vessel length

- $L_{c g i}$ is the distance from the center of gravity to the vessel bow

- $u_{i}$ is the surge speed of the vessel in its body frame

- $v_{i}$ is the sway speed in the body frame

- $\theta_{i}$ is the yaw angel in the Earth coordinate frame

- $\tau_{1}$ is the thrust generated by the prime mover

- $\tau_{3}$ is the yaw torque generated by the rudder system

- $\quad M_{i}$ is the inertial matrix for vessel $i$

- $D_{i}$ is the damping matrix for vessel $i$

- $C_{i}$ is the term representing the Coriolis and centrifugal forces.

The control inputs for this underactuated system are $\tau_{1}$ and $\tau_{3}$ which have to control three state variables of the system through nonlinear equations. These equations also consist nonholonomic constraints which bring limitation on velocities to be performed. Every vessel will be operating in displacement mode, so the weight of vessel is supported by buoyant force.

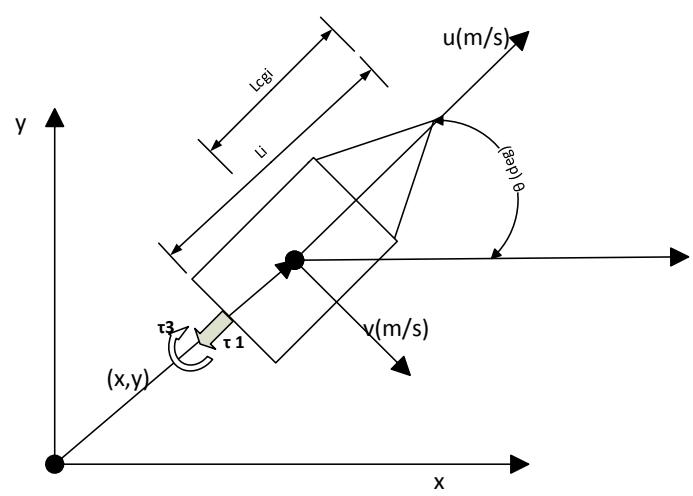

Figure 1. Autonomous underwater vehicle Model

\section{Mission Statement}

Considering $N$ underactuated surface vessels. During control mission, each vessel knows its own state and the states of some neighboring vessels by communication links [8]. This communication topology will be described by the aid of graph theory. It is assumed that all agents know their owns states and the states of the other vessels.

Given a desired geometric pattern $\mathrm{P}$ defined by constant vectors $\left[p_{j x}, p_{j y}\right]$ for $1 \leq j \leq n$, assuming:

$$
\sum_{j=1}^{n} p_{j x}=0, \quad \sum_{j=1}^{n} p_{j y}=0
$$

Based on the desired trajectory, designing a cooperative controller for each vessel such that [8]:

$$
\begin{gathered}
\lim _{t \rightarrow \infty}\left(\left[\begin{array}{l}
x_{i}-x_{j} \\
y_{i}-y_{j}
\end{array}\right]\right. \\
\left.-\left[\begin{array}{l}
p_{i x}-p_{j x} \\
p_{i y}-p_{j y}
\end{array}\right]\right)=\left[\begin{array}{l}
0 \\
0
\end{array}\right] \\
\lim _{t \rightarrow \infty}\left(\theta_{j}-\theta_{d}\right)=0 \\
\lim _{t \rightarrow \infty}\left(u_{j}-u_{d}\right)=0 \\
\lim _{t \rightarrow \infty}\left(v_{j}-v_{d}\right)=0 \\
\lim _{t \rightarrow \infty}\left(\dot{\theta}_{i}-\dot{\theta}_{d}\right)=0
\end{gathered}
$$

To achieve these control targets, coordination transfer is proposed here. Following the work [8], the new errors are defined base on system dynamics. The new errors are defined as: 


$$
\begin{aligned}
& \lim _{t \rightarrow \infty} e_{j} \\
& =\lim _{t \rightarrow \infty}\left(\left[\begin{array}{c}
x_{j} \\
y_{j} \\
\theta_{j} \\
u_{j} \\
v_{j} \\
\dot{\theta}_{j}
\end{array}\right]-\left[\begin{array}{c}
x_{d}+p_{j x} \\
y_{d}+p_{j y} \\
\theta_{d} \\
u_{d} \\
v_{d} \\
\dot{\theta}_{d}
\end{array}\right]\right) \\
& =0
\end{aligned}
$$

So the cooperative controller in between agents should be designed in such a way that the above control scheme to be achieved. Based on the agents' dynamic, new tracking errors are defined and the controller will be tuned based on these new errors. Tracking errors are defined as:

$$
\begin{aligned}
& \delta_{1 j}=\left(x_{j}-p_{j x}\right) \cos \left(\theta_{j}\right) \\
&+\left(y_{j}-p_{j y}\right) \sin \left(\theta_{j}\right) \\
&-\left(x_{d} \cos \left(\theta_{d}\right)\right. \\
&+y_{d} \sin \left(\theta_{d}\right) \\
& \delta_{2 j}= v_{j}-v_{d} \\
& \delta_{3 j}=-\left(x_{j}-p_{j x}\right) \sin \left(\theta_{j}\right) \\
&+\left(y_{j}-p_{j y}\right) \cos \left(\theta_{j}\right) \\
&+\frac{m_{2}}{d_{2}} v_{j} \\
&-\left(-x_{d} \sin \left(\theta_{d}\right)\right. \\
&+y_{d} \cos \left(\theta_{d}\right) \\
&\left.+\frac{m_{2}}{d_{2}} v_{d}\right) \\
& \delta_{4 j}=\theta_{j}-\theta_{d} \\
& \delta_{5 j}=\dot{\theta}_{j}-\dot{\theta}_{d}+k_{4} \delta_{4 j} \\
& \delta_{6 j}=-\frac{m_{1}}{d_{2}} u_{j}-\delta_{1 j}+\frac{m_{1}}{d_{2}} u_{d} \\
& \quad+k_{3} \delta_{5 d} \delta_{3 j}
\end{aligned}
$$

These new inputs coordinates are different from those three in the planar maneuver of any vessel [8]. These transfer coordinates are defined as:

$$
\begin{aligned}
w_{1 j}=\left(\frac{d_{1}}{d_{2}}-1\right) & u_{j}-\delta_{3 j} \delta_{6 j}-\frac{\tau_{1 j}}{d_{2}} \\
& -\left(-m_{32} \dot{v}_{d}\right. \\
& -\frac{d_{32}+c_{32}}{m_{32}} v_{d} \\
& \left.-\frac{c_{33}}{m_{33}} \dot{\theta}_{d}+\frac{\tau_{2 d}}{m_{33}}\right)
\end{aligned}
$$

$$
\begin{aligned}
w_{2 j}=-m_{32} \dot{v}_{j} & -\frac{d_{32}+c_{32}}{m_{32}} v_{j}-\frac{c_{33}}{m_{33}} \dot{\theta}_{j} \\
& +\frac{\tau_{2 j}}{m_{33}}-\left(\left(\frac{d_{1}}{d_{2}}-1\right) u_{d}\right. \\
& \left.-\delta_{3 d} \delta_{5 d}-\frac{\tau_{1 d}}{d_{2}}\right)
\end{aligned}
$$

The new errors are defined as $\delta_{*}=$ $\left[\delta_{1 j}, \delta_{2 j}, \delta_{3 j}, \delta_{4 j}, \delta_{5 j}, \delta_{6 j}\right]$. It is easy to show that if $\lim _{t \rightarrow \infty} \delta_{*}=0$, then $\lim _{t \rightarrow \infty} e=0$ achived. The dynamics of tracking errors are:

$$
\begin{aligned}
\dot{\delta}_{1 j}=-\frac{d_{2}}{m_{1}} \delta_{1 j} & -\frac{d_{2}}{m_{1}}\left(\delta_{6 j}-k_{3} \delta_{5 j} \delta_{3 j}\right) \\
& +\delta_{3 j}\left(\delta_{5 j}-k_{4} \delta_{4 j}\right) \\
& +\delta_{3 d}\left(\delta_{5 j}-k_{4} \delta_{4 j}\right) \\
& +\delta_{5 d} \delta_{3 j} \\
& -\frac{m_{2}}{d_{2}}\left[\delta_{2 j}\left(\delta_{5 j}-k_{4} \delta_{4 j}\right)\right. \\
& +\delta_{2 d}\left(\delta_{5 j}-k_{4} \delta_{4 j}\right) \\
& \left.+\delta_{2 j} \delta_{5 d}\right] \\
\dot{\delta}_{2 j}=-\frac{d_{2}}{m_{2}} \delta_{2 j} & +\frac{d_{2}}{m_{2}}\left[\delta_{1 j}\left(\delta_{5 j}-k_{4} \delta_{4 j}\right)\right. \\
& +\delta_{1 d}\left(\delta_{5 j}-k_{4} \delta_{4 j}\right) \\
& +\delta_{1 j} \delta_{5 d} \\
& +\left(\delta_{5 j}-k_{4} \delta_{4 j}\right)\left(\delta_{6 j}\right. \\
& \left.-k_{3} \delta_{5 d} \delta_{3 j}\right) \\
& +\left(\delta_{5 j}-k_{4} \delta_{4 j}\right) \delta_{6 d} \\
& \left.+\delta_{5 d}\left(\delta_{6 j}-k_{3} \delta_{5 d} \delta_{3 j}\right)\right] \\
\dot{\delta}_{6 j}=w_{2 j}-w_{2 d} & +k_{3} \dot{\delta}_{5 d} \delta_{3 j} \\
+ & k_{3} \delta_{5 d}\left(\delta_{5 j}-k_{4} \delta_{4 j}\right)\left(\delta_{6 j}\right. \\
- & \left.k_{3} \delta_{5 d} \delta_{3 j}\right) \\
+ & k_{3} \delta_{5 d}\left(\delta_{5 j}-k_{4} \delta_{4 j}\right) \delta_{6 d} \\
+ & k_{3} \delta_{5 d}^{2}\left(\delta_{6 j}-k_{3} \delta_{5 d} \delta_{3 j}\right) \\
\dot{\delta}_{3 j}=\left(\delta_{5 j}-k_{4}\right. & \left.\delta_{4 j}\right)\left(\delta_{6 j}-k_{3} \delta_{5 d} \delta_{3 j}\right) \\
& +\left(\delta_{5 j}-k_{4} \delta_{4 j}\right) \delta_{6 d} \\
& +\delta_{5 d}\left(\delta_{6 j}-k_{3} \delta_{5 d} \delta_{3 j}\right) \\
\dot{\delta}_{4 j}= & -k_{4} \delta_{4 j}+\delta_{5 j}-w_{1 d}+k_{4}\left(-k_{4} \delta_{4 j}+\delta_{5 j}\right) \\
&
\end{aligned}
$$

Based on the [8], following lemmas are addressed: Lemma 1: For the variables defined in the above formulas, if:

$$
\begin{aligned}
\lim _{t \rightarrow \infty}\left(\delta_{i j}-c_{i}\right)=0 \quad(1 \leq i \\
\leq 6, \quad 1 \leq j \leq m)
\end{aligned}
$$

Where $c_{i}$ bounded variables, then equation 9 are is 
being satisfied. Furthermore, if $c_{i}=0$ for $(1 \leq i \leq 6)$, then equations 9 to 13 are being satisfied.

Lemma 2: For system equations 15 to 20, if $\delta_{5 j}$ and $\delta_{6 j}$ exponentially converge to constants $c_{5}$ and $c_{6}$, respectively, for $1 \leq j \leq m$, then equation 9 is being satisfied. Furthermore, if $c_{5}=c_{6}=0$, then equations 9 to 13 are being satisfied, which means that the cooperative control problem is achieved.

\section{Communication Digraph}

Assumption 1: The communication digraph $G$ is fixed and has a spanning tree.

Given any $m * m$ constant matrix $A=\left[a_{j i}\right]$ with $a_{j i}>$ 0 for $1 \leq i, j \leq m$, the Laplacian matrix $L=\left[L_{j i}\right]$ of the digraph $\mathrm{G}$ with weight matrix $\mathrm{A}$ is defined as:

$$
\begin{aligned}
& L_{j i} \\
& =\left\{\begin{array}{lr}
-a_{j i} & \text { if } i \neq j \text { and } i \in N_{j} \\
0 & \text { if } i \neq j \text { and } i \in N_{j} \\
\sum_{l \neq j, l \in N_{j}} a_{j l} & \text { if } i=j
\end{array}\right.
\end{aligned}
$$

Lemma 3: $\mathrm{L}$ is the Laplacian matrix of the digraph $\mathrm{G}$ with weight matrix $A=\left[a_{j i}\right]$ and $a_{j i}>0$. If the digraph $\mathrm{G}$ satisfies Assumption 1, then:

$$
\begin{aligned}
\lim _{t \rightarrow \infty} e^{\mu t}\left(e^{-L t}-1 w_{l}^{T}\right) & \\
= & 0 \lim _{t \rightarrow \infty} e^{\mu t}\left(e^{-L t}\right. \\
- & \left.1 w_{l}^{T}\right)=0
\end{aligned}
$$

For any $\mu \in\left[0, \operatorname{Re}\left(\lambda_{2}(L)\right)\right]$, where $w_{l}$ satisfies $w_{l}^{T} L=0$ and $w_{l}^{T} 1=1$. accordingly [10].

\section{Cooperative Control Law}

For the systems in equations 1 to 7 , regarding to Lemma 1, 2 and 3 and assumption 1, control laws are:

$$
\begin{aligned}
\tau_{1 j}=\sum_{i} d_{2} a_{j i} & \left(\delta_{5 j}-\delta_{5 i}\right)-d_{2} w_{1 d} \\
& +d_{2} k_{4}\left(-k_{4} \delta_{4 j}+\delta_{5 j}\right) \\
& +\left(d_{1}-d_{2}\right) u_{j}-d_{2} \delta_{3 j} \delta_{6 j} \\
& -d_{2}\left(-m_{32} \dot{v}_{d}\right. \\
& -\frac{d_{32}+c_{32}}{m_{32}} v_{d}-\frac{c_{33}}{m_{33}} \dot{\theta}_{d} \\
& \left.+\frac{\tau_{2 d}}{m_{33}}\right)
\end{aligned}
$$

$$
\begin{aligned}
\tau_{2 j}=-\sum_{i} m_{3} & a_{j i}\left(\delta_{6 j}-\delta_{6 i}\right)+m_{3} w_{2 d} \\
& -m_{3} k_{3} \dot{\delta}_{5 d} \delta_{3 j} \\
& -m_{3} k_{3} \delta_{5 d}\left(\delta_{5 j}\right. \\
& \left.-k_{4} \delta_{4 j}\right)\left(\delta_{6 j}-k_{3} \delta_{5 d} \delta_{3 j}\right) \\
& -m_{3} k_{3} \delta_{5 d}\left(\delta_{5 j}\right. \\
& \left.-k_{4} \delta_{4 j}\right) \delta_{6 d} \\
& -m_{3} k_{3} \delta^{2}{ }_{5 d}\left(\delta_{6 j}\right. \\
& \left.-k_{3} \delta_{5 d} \delta_{3 j}\right) \\
& -\left(m_{1}-m_{2}\right) u_{j} v_{j}+d_{3} \dot{\theta}_{j} \\
& +m_{3}\left(\left(\frac{d_{1}}{d_{2}}-1\right) u_{d}-\delta_{3 d} \delta_{5 d}\right. \\
& \left.-\frac{\tau_{1 d}}{d_{2}}\right)
\end{aligned}
$$

Which $a_{j i}>0, k_{3}>0$, and $k_{4}>0$.

Group of underwater vessels approaches the desired pattern by the control laws in equation 32 and equation 33. Besides, these control laws have decentralized and scalable properties while performing proceed. Simulation results in [8] are used for small crafts and vessels with very simple dynamics, while proposed approach here has more generality.

\section{Simulation Results}

The performance of the control laws is shown in following figures, tables, and discussions. In this research work, 3 underwater vehicles which their basic dynamic parameters were adopted from [9], are under control to converge to the desired pattern. The communication digraph is assumed to be fixed. Control parameters are as $a_{i j}=1.3, k_{3}=$ 25 and $k_{4}=25$. Initial conditions corresponding to the vessels are given in table 1 .

Table 1. Initial Condition of 3 underwater vessels

\begin{tabular}{cc}
\hline$\left[x_{1}(0), x_{2}(0), x_{3}(0)\right]$ & {$[10,10,15][\mathrm{m}]$} \\
\hline$\left[y_{1}(0), y_{2}(0), y_{3}(0)\right]$ & {$[40,23,10][\mathrm{m}]$} \\
\hline$\left[\theta_{1}(0), \theta_{2}(0), \theta_{3}(0)\right]$ & {$\left[\frac{\pi}{4}, \frac{\pi}{4}, 0\right][\mathrm{rad}]$} \\
\hline$\left[u_{1}(0), u_{2}(0), u_{3}(0)\right]$ & {$[0,0,0][\mathrm{m} / \mathrm{s}]$} \\
\hline$\left[v_{1}(0), v_{2}(0), v_{3}(0)\right]$ & {$[0,0,0][\mathrm{m} / \mathrm{s}]$} \\
\hline$\left[r_{1}(0), r_{2}(0), r_{3}(0)\right]$ & {$[0,0,0][\mathrm{rad} / \mathrm{s}]$} \\
\hline
\end{tabular}

Figure 2 demonstrates the entire formation trajectories of the systems. Figure 3 and 4 show that the control efforts with surge force and steering torque are bounded. Consequently, figures 3 and 4 confirms that the level of control activity is reasonable, and no saturation has occurred during the process. The decreasing trend of the trajectory error of the system in the first scenario is shown in Figure 5. Also, in 
figure 6, Collective Movement of three underwater robots is presented to prove the non-collision behaviour and cooperation between the agents. Figure 7 shows the trajectory path of the five agents. It demonstrates the scalability of the system.

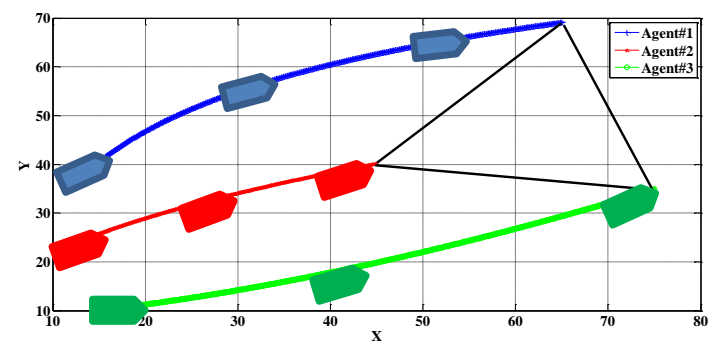

Figure 2. Trajectory Formation of the system

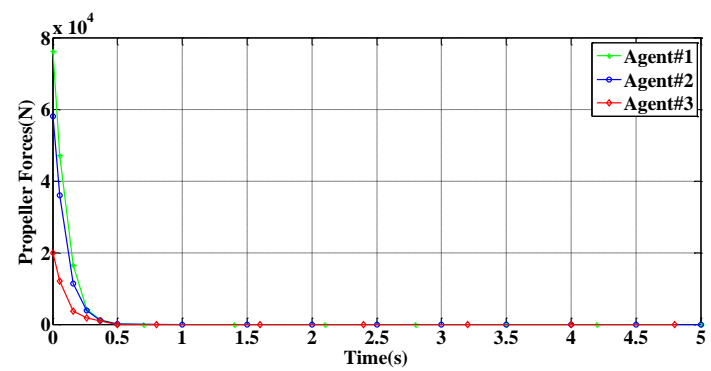

Figure 3. Propeller control Force of the agents

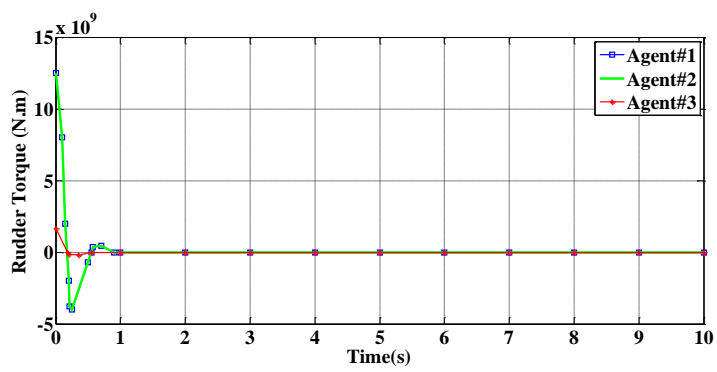

Figure 4. Rudder torque of the Agents

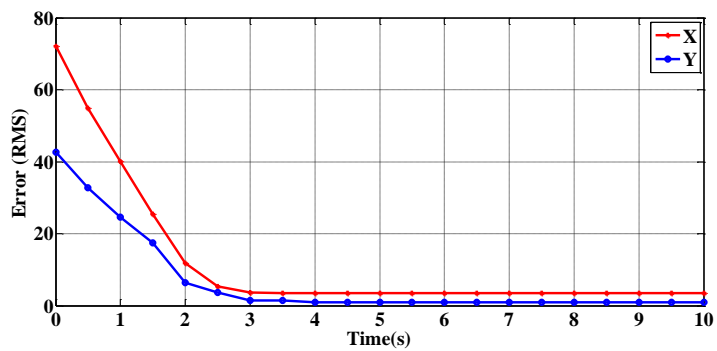

Figure 5. Time history of the system error (RMS)

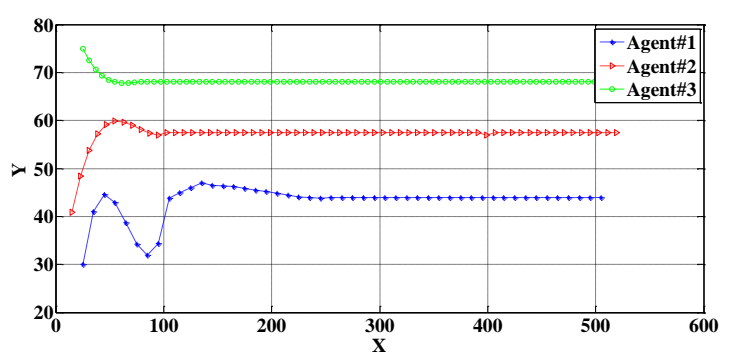

Figure 6. Collective movement of the Agents

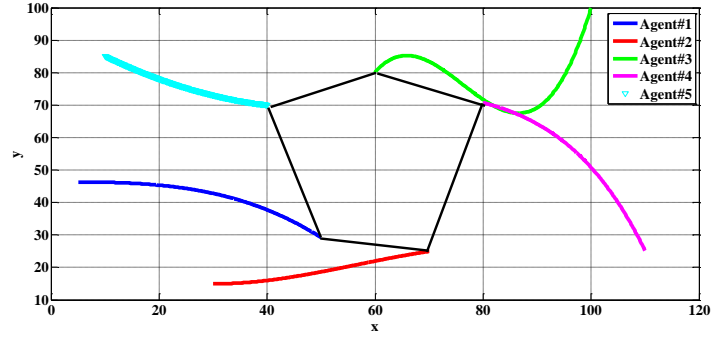

Figure 7. Trajectory formation of the 5 agents

\section{Conclusions}

In this project, we have tried to use the potential functions of the Lyapunov and graph theory to control a group of the underwater collaborators for a particular formation. In this project the actual dynamics of the underwater robots have been used because of the complexities and non- Holonomic properties of this dynamic to control, a coordination transfer is used. The lack of external monitoring and external control are the most important advantages of this project compared to previous work. formation maneuvering for a specific shape has been performed for two groups of three and five. According to the presented results, the designed controller responds well to the all needed .Another item in the co-op robots is collective movements, as well as no collision happens with each other in different maneuvers, which has been accomplished by simulating a triple group for this purpose. As the results show, the controller is well-responsive and can be used in group work. The designed controller could easily be applied to the underwater vessels by the mean of Industrial Computer, and I prefer it for future work in this regard. Also, this controller can be extended to a different kind of Non-Holonomic Dynamic such as Motor vehicles.

\section{List of Symbols}

$\begin{array}{cl}\mathrm{C} & \text { Coriolis matrix } \\ \mathrm{D} & \text { Damping matrix } \\ \mathrm{e} & \text { Error } \\ \mathrm{L} & \text { Length } \\ \mathrm{M} & \text { Inertial matrix } \\ \tau_{1} & \text { Surge force } \\ \tau_{2} & \text { Steering torque } \\ \mathrm{u} & \text { Surge speed } \\ \mathrm{x} & \text { Longitudinal position } \\ \mathrm{y} & \text { Vertical position } \\ \theta & \text { Yaw } \\ \delta & \text { Tracking error }\end{array}$

\section{References}

1-Fiorelli, N. E. L. a. E., (2001), Virtual leaders, artificial potentials and coordinated control of groups, Decision and Control. Orlando, FL, USA, USA, IEEE. [ DOI:10.1109/CDC.2001.980728]

2-Filippo Arrichiello., S. C., Thor I. Fossen, (2006), Formation Control of Underactuated Surface Vessels using the Null-Space-Based Behavioral Control, 
Intelligent Robots and Systems. Beijing, China IEEE. [ DOI:10.1109/IROS.2006.282477]

3- Jagannathan, T. D. a. S., (2007), Control of Nonholonomic Mobile Robot Formations: Backstepping Kinematics into Dynamics. IEEE International Conference on Control Applications. Singapore, Singapore, IEEE. [ DOI:10.1109/CCA.2007.4389212].

4- Hashem Ashrafiuon, K. R. M., Lucas C. McNinch (2008), Sliding-Mode Tracking Control of Surface Vessels, IEEE Transactions on Industrial Electronics 55. [DOI: 10.1109/ACC.2008.4586550].

5- Jevtic, A., Gazi, P., Andina, D., Jamshidi, M., (2010), Building a swarm of robotic bees, World Automation Congress (WAC).

6- Barış Fidan, V. G., Shaohao Zhai, (2013), SingleView Distance-Estimation-Based Formation Control of Robotic Swarms, IEEE Transactions on Industrial Electronics 60(12).

[DOI:10.1109/TIE.2012.2236996].

7- Gazi, V., (2014), Distributed output agreement in a class of uncertain linear heterogeneous multi-agent dynamic systems, Control Conference (ECC). Strasbourg, France, IEEE. [DOI:10.1109/ECC.2014.6862575].

8- Dong, W., (2010), Cooperative control of underactuated surface vessels, IET Control Theory \& Applications 4(9). [ DOI:10.1049/iet-cta.2009.0362].

9- Bishop, B. E., (2012), Formation control of underactuated autonomous surface vessels using redundant manipulator analogs, IEEE International Conference on Robotics and Automation (ICRA), Saint Paul, MN, USA IEEE. [DOI: 10.1109/ICRA.2012.6224865].

10- I.-A.F. Ihle, J. J., T.I. Fossen, (2006), Robust Formation Control of Marine Craft Using Lagrange Multipliers, Springer, Berlin, Heidelberg. [DOI: 10.1007/11505532_7].

11- Jan Tommy Gravdahl, K. Y. P., Henk Nijmeijer, (2006), Group Coordination and Cooperative Control, Berlin, Germany, Springer-Verlag Berlin and Heidelberg GmbH \& Co. KG [DOI:10.1007/11505532].
12- Gazi, V., Passino, Kevin M., (2011), Swarm Stability and Optimization, springer. [DOI:10.1007/978-3-642-18041-5].

13- Chung, F. R. K., (1997), Spectral Graph Theory, AMS and CBMS.

14- RussellMerris, (1998), Laplacian graph eigenvectors, Linear Algebra and its Applications 278: 1-7.

15- W. Dong, J. A. F., (2008), Formation control of multiple underactuated surface vessels, IET Control Theory \& Applications 2(12). [ DOI:10.1049/ietcta:20080183].

16- Lucas C. McNinch, H. A., (2011), Predictive and sliding mode cascade control for Unmanned Surface Vessels, American Control Conference (ACC), San Francisco, CA, USA IEEE. [DOI:10.1109/ACC.2011.5991049].

17- Arkin, R. C., (1998), Behavior-Based Robotics MIT Press.

18- Brooks, R., (1986), A robust layered control system for a mobile robot, IEEE Robotics and Automation Society.

19- Arkin, R., (1989), Motor schema-based mobile robot navigation, The International journal of robotics research. [DOI:10.1109/ROBOT.1987.1088037].

20- Krishnaprasad, E. W. J. a. P. S., (2004), Equilibrium and steering laws for planar formations, IEEE Trans. Robot. Automat 19. [DOI:10.1016/j.sysconle.2003.10.004].

21- J.R.T. Lawton, R. W. B., B.J. Young, (2003), A decentralized approach to formation maneuvers, IEEE Robotics and Automation Society 19(6). [DOI:10.1109/TRA.2003.819598].

22- Joshua A. Marshall, M. E. B., Bruce A. Francis (2006), Pursuit formations of unicycles, Automatica (Journal of IFAC) 42(1). [DOI:10.1016/j.automatica.2005.08.001].

23- Zhiyun Lin, B. F., M. Maggiore, (2005), Necessary and sufficient graphical conditions for formation control of unicycles, IEEE Control Systems Society 50(1). [DOI:10.1109/TAC.2004.841121]. 